\title{
Physical Layer Security Scheme Based on Polarization Modulation and WFRFT Processing for Dual-polarized Satellite Systems
}

\author{
Zhangkai Luo $^{1 *}$, Huali Wang ${ }^{1}$, Kaijie Zhou ${ }^{1}$ \\ ${ }^{1}$ College of Communications Engineering, PLA Army Engineering University, \\ Nanjing 210007, CHINA \\ [e-mail: luo_zhangkai@126.com, huali.wang@ieee.org, 530056812@qq.com] \\ *Corresponding author: Zhangkai Luo
}

Received May 6, 2017; revised June 25, 2017; accepted July 24, 2017;

published November 30, 2017

\begin{abstract}
A novel scheme based on polarization modulation and the weighted fractional Fourier transform (PM-WFRFT) is proposed in this paper to enhance the physical layer security of dual-polarized satellite systems. This scheme utilizes the amplitude and phase of the carrier as information-bearing parameters to transmit the normal signal and conceals the confidential information in the carrier's polarization state (PS). After being processed by WFRFT, the characteristics of the transmit signal (including amplitude, phase and polarization state) vary randomly and in nearly Gaussian distribution. This makes the signal very difficult for an eavesdropper to recognize or capture. The WFRFT parameter is also encrypted by a pseudo-random sequence and updated in real time, which enhances its anti-interception performance. Furthermore, to prevent the polarization-based impairment to PM-WFRFT caused by depolarization in the wireless channel, two components of the polarized signal are transmitted respectively in two symbol periods; this prevents any mutual interference between the two orthogonally polarized components. Demodulation performance in the system was also assessed, then the proposed scheme was validated with a simulated dual-polarized satellite system.
\end{abstract}

Keywords: Polarization modulation, WFRFT, physical layer security, dual-polarized satellite 


\section{Introduction}

Security is a fundamental problem in satellite systems due to the open nature of the wireless medium and its wide beam coverage, which makes it difficult to shield transmitted signals from unintended recipients [1]. Transmission security is traditionally dependent on cryptographic techniques in the link and network layer. Challenges (and vulnerabilities) associated with key distribution and management, however, make cryptography a less than ideal solution. New research on physical layer security has focused primarily on guaranteeing communication signals with low probability of interception (LPI) and low probability of detection (LPD).

There currently exist several anti-detection techniques for improving the LPD performance of communication signals, such as adaptive beamforming [2], spread-spectrum techniques [3, 4], and frequency hopping techniques [5]. Although these techniques can make it hard for an eavesdropper to detect the target signal, safety can not be guaranteed once the signal is captured. On the other hand, researchers have focused on two main approaches in attempting to improve the LPI performance. The first approach thinks that the transmission security can be realized if the legitimate user's channel is better than that of the eavesdropper [6]. Thus the techniques typically aims to enhance the security capacity, such as adding precoding techniques or artificial noise [1, 7-9]. The other approach is to deteriorate the eavesdropper's channel quality, for instance, through direction modulation [10,11] or physical layer encryption techniques [12], the constellation structure of the signal is effectively distorted, which make it difficult for the eavesdropper to demodulate any useful information. These techniques can protect the target signal, but the signal can still easily be detected, the system is vulnerable to jamming attacks [13].

In satellite systems, precoding techniques based on the channel state information (CSI) at the transmitter side are difficult to implement due to the lengthy round trip between the satellite and the receiver, since the CSI has changed by the time the transmitter receives the CSI feedback. Moreover, the CSI of the eavesdropper (especially a passive one) is hard to obtain in practice. Therefore, secure transmission techniques that do not require CSI have grown increasingly popular, to this effect. Further, satellite scenarios make the spatial components become correlated at the receiver side due to the Line of Sight (LOS), and without sufficient scattering, the receiver can only discover a single transmission path because it does not have sufficient sensitivity to distinguish the different spatial signatures. So if the eavesdropper is close to the legitimate user, the method like in [14] will not perform well, which is based on the channel difference between the two parties. Under this condition, polarization diversity is a suitable choice for the satellite scenarios [15], which can improve the transmission efficiency [16]. However, in dual-polarized satellite systems, the security problem receives little attention and there is also no extra degree for designing artificial noise to improve the security rate. In addition, precoding techniques are also improper. Thus, to improve the transmission security performance is very challenging.

In this study, we explored both LPI and LDP characteristics of transmit signals and built a physical layer security scheme for the dual-polarized satellite system based on polarization modulation (PM) and weighted fractional Fourier transform (PM-WFRFT) techniques [17]. PM is a three-dimensional modulation technique based on dual-polarized antennas, which utilizes the polarization state (PS) as the information-bearing parameter [18]. Interestingly, there is no contradictions when PM is combined with traditional modulation techniques (e.g., 
MPSK, MQAM, FSK) which utilize the amplitude, phase, and frequency as information-bearing parameters. Therefore, in the PM-WFRFT scheme, the traditional modulated signal carries the unclassified information while the confidential information is concealed in its polarization state. After WFRFT processing, the time-frequency characteristics of the polarized signal vary randomly and its distribution is nearly Gaussian, which make it very difficult to be detected. In addition, the WFRFT parameter in the proposed scheme is controlled by a pseudo-random sequence and updated in real time to guarantee favorable LPI performance.

Authors in [19] analyzed the impairment to the polarization-based channel, which we refer to in this paper as the polarization-dependent loss (PDL) effect. To address this problem, we apply the spatial modulation (SM) concept to dual-polarized communications [20], where two components of the polarized signal are transmitted sequentially in two symbol periods, so that there is no mutual interference between the two orthogonally polarized components. Moreover, there is no CSI requirement at the transmitter side. We also propose a simple and effective method for distinguishing the polarization characteristics of the transmitting antenna. Our simulation results show that the PM-WFRFT scheme can be applied conveniently to traditional satellite systems. Notably, although the proposed scheme is designed for satellite scenarios, it can also be appropriately changed to be applied in other wireless communication systems such as MIMO systems [21] [22] and relaying networks [23, 24].

\section{System Model}

\subsection{Principle of the PM-WFRFT Scheme}

To provide relevant details about the PM-WFRFT scheme, we provided a block diagram of the transmitter in Fig.1. The information sequence contains two parts: unclassified information $\mathrm{I}_{q}$, $\mathrm{I}_{b}$ and confidential information $\mathrm{I}_{p}$. As shown in Fig. 1, $\mathrm{I}_{q}$ is modulated by the traditional modulation (TM) technique, which yields constellation points $\left\{Q_{k}\right\}_{k=1}^{K}$ :

$$
Q_{k}=A_{k} e^{j \phi_{k}}, k=1,2, \cdots, K,
$$

where $A_{k}$ and $\phi_{k}$ denote the anplitude and the phase of the $k$-th symbol. $Q_{k}$ is then duplicated for further processing.

Confidential information $\mathrm{I}_{p}$ is mapped to a group of PM constellation points by polarization modulation, which yields constellation points $\left\{P_{k}\right\}_{k=1}^{K} \in\left\{P_{i}\right\}_{i=1}^{M_{P}}$, where $M_{P}$ is the modulation order. The constellation points can then be mapped into weighted values $\left\{\mathbf{w}_{k}\right\}_{k=1}^{K}$ :

$$
\mathbf{w}_{k}=\left[\begin{array}{l}
w_{1}^{k} \\
w_{2}^{k}
\end{array}\right]=\left[\begin{array}{l}
1 \\
\tan \gamma_{k} e^{j \eta_{k}}
\end{array}\right], k=1,2, \cdots, K,
$$

where $\gamma_{k} \in\left[0, \frac{\pi}{2}\right], \eta_{k} \in[0,2 \pi]$. Next, each duplicated $Q_{k}$ is multiplied by $w_{m}^{k}, m=1,2$, in Eq. (2) and the results are:

$$
\begin{aligned}
& s_{1}^{k}=A_{k} w_{1}^{k} e^{j \phi_{k}}=A_{k} e^{j \phi_{k}} \\
& s_{2}^{k}=A_{k} w_{2}^{k} e^{j \phi_{k}}=A_{k} \tan \gamma_{k} e^{j \eta_{k}} e^{j \phi_{k}},
\end{aligned}
$$

Two branch signals are then processed by WFRFT to yield WFRFT signals: 


$$
\begin{aligned}
& \mathbf{s}_{1}^{\mathrm{W}}=\mathcal{F}^{a_{1}}\left[\mathbf{s}_{1}\right], \\
& \mathbf{s}_{2}^{\mathrm{W}}=\mathcal{F}^{a_{2}}\left[\mathbf{s}_{2}\right],
\end{aligned}
$$

where $a_{1}$ and $a_{2}$ are transform parameters, $\mathbf{s}_{m}=\left[s_{m}^{1}, s_{m}^{2}, \cdots, s_{m}^{K}\right]^{\mathrm{T}}, m=1,2 \cdot \mathbf{s}_{1}^{\mathrm{W}}$ and $\mathbf{s}_{2}^{\mathrm{W}}$ are then passed through a digital-to-analog converter, up converter, and sent to the polarization selection (POS) unit. The transmit signals can be denoted as follows:

$$
\begin{aligned}
& \mathbf{s}_{1}^{\mathrm{s}}=\mathcal{F}^{a_{1}}\left[\mathbf{s}_{1}\right] e^{j \mathrm{w}_{c} t} \\
& \mathbf{s}_{2}^{\mathrm{s}}=\mathcal{F}^{a_{2}}\left[\mathbf{s}_{2}\right] e^{\mathrm{j} \mathrm{w}_{c} t},
\end{aligned}
$$

where $w_{c}$ is the carry frequency. Finally, during two symbol periods, they are transmitted in turn by the horizontal- or vertical-polarized antennna according to $\mathbf{I}_{b}:\left\{b_{1}^{k} b_{2}^{k}\right\}_{k=1}^{K}$.

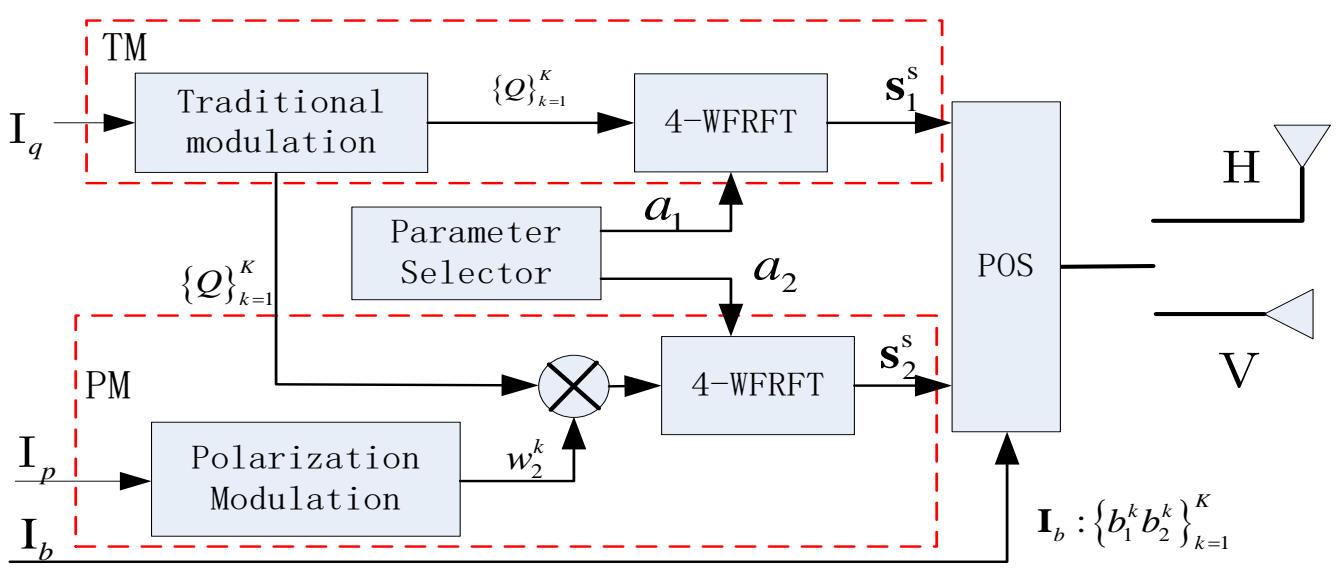

Fig. 1. Block diagram of the transmitter

Table 1 shows the relationship between the bit $b$ and the polarization of the transmitting antenna. If $\left\{b_{k}^{1} b_{k}^{2}\right\}=\{10\}$, for example, $s_{1}^{s}(k)$ is transmitted by horizontally-polarized antenna $(\mathrm{H})$ in the first symbol period and $s_{2}^{s}(k)$ is transmitted by vertically-polarized antenna $(\mathrm{V})$ in the second symbol period for the $k$-th constellation point.

Table 1. The relationship between the bit $b$ and the polarization of the transmitting antenna

\begin{tabular}{|c|c|c|c|c|}
\hline$\left\{b_{1}^{k} b_{2}^{k}\right\}$ & $\{00\}$ & $\{01\}$ & $\{10\}$ & $\{11\}$ \\
\hline$s_{1}^{s}(k)$ & $\mathrm{V}$ & $\mathrm{V}$ & $\mathrm{H}$ & $\mathrm{H}$ \\
\hline$s_{2}^{s}(k)$ & $\mathrm{V}$ & $\mathrm{H}$ & $\mathrm{V}$ & $\mathrm{H}$ \\
\hline
\end{tabular}

\subsection{Weighted Fractional Fourier Transform}

Let $\mathbf{I}_{N}$ be the $N \times N$ identity matrix and $\mathbf{F}_{N}$ denotes the unitary Fourier matrix. The shift matrix $\mathbf{P}_{N}$ can be represented as follows [25]: 


$$
\mathbf{P}_{K}=\left[\begin{array}{cccccc}
1 & 0 & \cdots & 0 & 0 & 0 \\
0 & 0 & \cdots & 0 & 0 & 1 \\
0 & 0 & \cdots & 0 & 1 & 0 \\
\vdots & \vdots & 0 & \ddots & 0 & 0 \\
0 & 0 & 1 & 0 & \cdots & 0 \\
0 & 1 & 0 & 0 & \cdots & 0
\end{array}\right]_{K \times K},
$$

According to the properties of the Fourier matrix, $\mathbf{F}_{K}^{-1}=\mathbf{F}_{K}^{H}=\mathbf{P}_{K} \mathbf{F}_{K}$. The $K \times K$ matrix is :

$$
\begin{aligned}
\mathbf{W}_{K}(a) & =w_{0}(a) \mathrm{I}_{K}+w_{1}(a) \mathbf{F}_{K}+w_{2}(a) \mathbf{P}_{K}+w_{3}(a) \mathbf{F}_{K}^{-1} \\
& =w_{0}(a) \mathrm{I}_{K}+w_{1}(a) \mathbf{F}_{K}+w_{2}(a) \mathbf{P}_{K}+w_{3}(a) \mathbf{P F}_{K}
\end{aligned},
$$

where $w_{l}(a), l=1,2,3,4$, denotes the weighting coefficient, which can be calculated as follows:

$$
w_{l}(a)=\frac{1}{4} \sum_{g=1}^{4} \exp \left[\frac{\mathrm{j} 2 \pi g(a-l)}{4}\right], l=1,2,3,4 .
$$

If $a$ is a real number, then $w_{l}(a)=w_{l}(a+4)$, thus, $a \in[0,4]$ or $a \in[-2,2]$. The transmit signal can be calculated as follows :

$$
\begin{aligned}
& \mathbf{s}_{1}^{\mathrm{s}}=\mathbf{W}_{K}\left(a_{1}\right) \mathbf{s}_{1} e^{j w_{c} t} \\
& \mathbf{s}_{2}^{\mathrm{s}}=\mathbf{W}_{K}\left(a_{2}\right) \mathbf{s}_{2} e^{\mathrm{j} w_{c} t},
\end{aligned}
$$

where $a_{1}$ and $a_{2}$ are parameters of traditional modulation branch (TM) and polarization modulation branch (PM) signals, respectively.

\section{Security Performance Evaluation}

\subsection{Power Spectral Density Analysis and PM Constellation Design}

Spectral characteristics are critical for successfully identifying target communication signals, such as central frequency, bandwidth, and spectral shape [3, 14]. In order to conceal the confidential information in the carrier's PS, the spectral characteristics of the traditional modulated signal must remain unchanged after PM is performed. Based on Eq. (3), the modulated information sequences can be denoted as follows:

$$
\left\{s_{m}^{n}\right\}=\sum_{n=-\infty}^{\infty} p_{m}^{n} q_{m}^{n} \delta(t-n T), m=1,2
$$

where $p_{m}^{n}=w_{m}^{n}, q_{m}^{n}=A_{n} e^{j \phi_{n}}$ and $T$ is the symbol period. The low pass signal can be denoted as

$$
v_{\text {low }}^{m}(t)=\sum_{n=-\infty}^{\infty} s_{m}^{n} g(t-n T),
$$

where $g(t)$ denotes the impulse response of pulse forming filter and its transfer function is $G(f)$. Then the PSD can be denoted as follows:

$$
\begin{aligned}
& S_{v, m}(f)=\frac{1}{T}|G(f)|^{2} \sum_{n=-\infty}^{\infty} R_{s, m}(n) e^{-j 2 \pi k f T}=\frac{1}{T}|G(f)|^{2} S_{s, m}(f) \\
& S_{s, m}(f)=\sum_{n=-\infty}^{\infty} R_{s, m}(n) e^{-j 2 \pi k f T} \\
& R_{s, m}(n)=E\left[s_{m}^{n+z} s_{m}^{n}\right]=E\left[p_{m}^{n+z} p_{m}^{n}\right] E\left[q_{m}^{n+z} q_{m}^{n}\right]
\end{aligned}
$$


where $R_{s, m}(n)$ is the autocorrelative function, $S_{v, m}(f)$ can be further expressed as follows [26]

$$
\begin{aligned}
S_{v, m}(f)= & \frac{E\left[s_{m}^{n}\right]^{2}}{T} \sum_{n=-\infty}^{\infty}\left|G\left(\frac{n}{T}\right)\right|^{2} \delta\left(f-\frac{n}{T}\right) \\
& +\frac{E\left[s_{m}^{n}\right]^{2}+E\left[\left(s_{m}^{n}\right)^{2}\right]}{T}|G(f)|^{2}
\end{aligned}
$$

where Eq. (12a) represents the discrete spectrum, the frequency scale of which is $\frac{1}{T}$ and Eq. (12b) represents the continuous spectrum. In the PM-WFRFT scheme, both the PM symbols and the traditional modulation symbols are statistically independent, so:

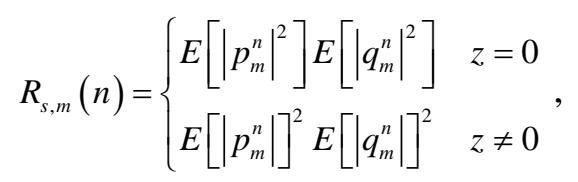

Based on Eq. (13) and Eq. (14), in order to avoid the discrete spectrum, the mean of the traditional modulation symbols should be equal to 0 . The PSD is then deteimined by $E\left[\left(s_{m}^{n}\right)^{2}\right]$ and $G(f)$. Therefore, The requirement for designing the PM constellation is

$$
\min \Lambda=\left|E\left[\left|p_{2}^{n}\right|^{2}\right]-1\right| \text {, }
$$

Of course, $\Lambda=0$ and $\left|E\left[\left|\tan \gamma_{n} e^{j \eta_{n}}\right|^{2}\right]=1\right| \Rightarrow \gamma_{n}=\frac{\pi}{4}$ is the optimal design. Therefore, the constellation points are on the same large circle on the Poincare ball, which is perpendicular to the $g_{1}$ axis as shown in Fig. 2. Each point on the Poincare sphere can be described by $\left(\gamma_{n}, \eta_{n}, \varphi_{n}, t_{n}\right)$, where $2 \gamma_{n}$ and $\eta_{n}$ denote the length of the arc from $P_{M}$ to the horizontal PS $P_{\mathrm{H}}$ and the angle between this arc and the equator respectively. $\varphi_{n}$ and $t_{n}$ are co-latitude and azimuth of $P_{M}$. The transition of the transmitted polarization states is continuous, and according to $[14,27]$, the produced PSD has narrower main lobe and larger side lobe attenuation compared with the traditional modulation method with the same simulation condition. Therefore, under said conditions, the spectral characteristics do not change.

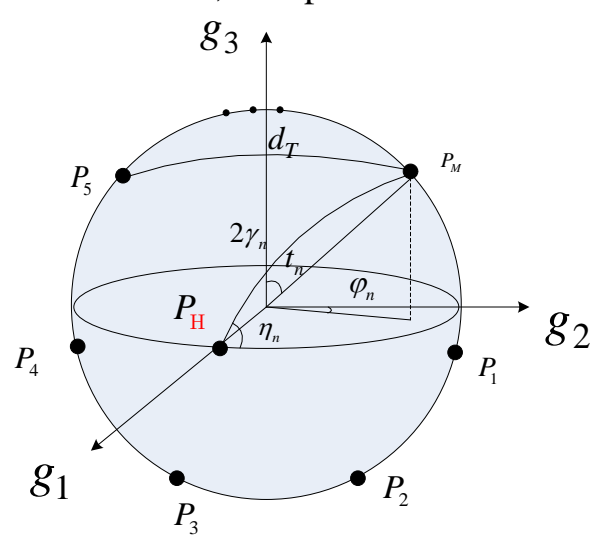

Fig. 2. PM constellation structure 


\subsection{Low Probability of Detection}

The closer the distribution of the transmit signal to Gaussian statistics, the harder it is to be detected [17]. That is because a quasi-Gaussian signal is so similar to noise that it is simply too challenging for eavesdroppers to recognize or capture. Signal detection or modulation recognition methods based on higher order cumulants (HOCs) are popular currently, not only for their favorable recognition capability, but also they are immune to AWGN as the HOCs of Gaussian functions are equal to zero, which is the main reason why these methods are not suitable for detecting quasi-Gaussian signals. We explored the similarity between the WFRFT signal and Gaussian signal as the discussion below.

Based on Eq. (4) and Eq. (7), the WFRFT signal can be rewritten as follows:

$$
\begin{aligned}
& \mathbf{W}_{K}\left(a_{m}\right) \mathbf{s}_{m}=\mathbf{X}_{m}^{1}+\mathbf{X}_{m}^{2}+\mathbf{X}_{m}^{3}+\mathbf{X}_{m}^{4} \\
& =w_{0}\left(a_{m}\right) \mathbf{s}_{m}+w_{1}\left(a_{m}\right) \mathbf{F}_{K} \mathbf{s}_{m}+w_{2}\left(a_{m}\right) \mathbf{P}_{K} \mathbf{s}_{m}+w_{3}\left(a_{m}\right) \mathbf{P} \mathbf{F}_{K} \mathbf{s}_{m},
\end{aligned}
$$

As the mean of $\mathbf{s}_{m}$ is $\mu=0$ and the variance is $\sigma^{2}$, we obtain:

$$
E\left[\mathbf{X}_{m}^{1}\right]=E\left[\mathbf{X}_{m}^{3}\right]=0,
$$

Additionally, because both the PM and TM constellation points are symmetrical to the original point, the constellation points of their combination are also symmetric to the original point. Moreover, the conbined signles are independent identically distributed (i.i.d.) and the real and imaginary parts of each complex symbol are independent of each other as well. As a result, from the central limit theorem, $\mathbf{X}_{m}^{2}$ and $\mathbf{X}_{m}^{4}$ obey Gaussian probability distribution with the mean $\mu$.

$$
\mu=E\left[\mathbf{X}_{m}^{2}\right]=E\left[\mathbf{X}_{m}^{4}\right]=0,
$$

So, based on Eq. (17) and Eq. (18), we derive $E\left[\mathbf{W}_{K}\left(a_{m}\right) \mathbf{s}_{m}\right]=0$. Notablely, WFRFT is a kind of unitary transformation, it satisfies the law of conversation of energy. Therefore, the variances $\sigma^{2}$ keep steady, which are the representations of signal's energy.

According to Eq. (16), the distribution of the WFRFT signal is affected by the WFRFT parameter. As authors in [17] point out that, as the parameter $a$ changes from an even number to an odd number, the distribution of the WFRFT signal changes from rule-based to Gaussian. Therefore, an appropriate $a$ value brings the WFRFT signal closer to Gaussian. In the PM-WFRFT scheme, $\mathbf{s}_{m}$ is the combination of the PM signal and the TM signal, and has total modulation order greater than the TM signal alone. Therefore, its distribution would be closer to Gaussian statistics [17]. The definition of Kurtosis is given to classify the signals to measure the similarity between the WRFRT and Gaussian signals:

$$
J=\frac{E\left[\mathbf{s}_{i}^{4}\right]}{E\left[\mathbf{s}_{i}^{2}\right]^{2}}-3,
$$

If $J=0$, the signal is Gaussian; if $J<0$, the signal is sub-Gaussian; If $J>0$, it is super-Gaussian.

\subsection{Low Probability of Interception}

We considered a worst-case scenario in which the eavesdropper knows the confidential information is concealed in the PS of the TM signal and it has been processed by WFRFT. Once the signal is captured, the eavesdropper must scan the entire range of parameters to find the WFRFT parameter $a$. Here, the QPSK signal which is processed by WFRFT was used as 
the TM signal. We assumed $a=0.7$ and set the scan step $\varepsilon$ to $0.1,0.01,0.05$ to observe the BER performance versus SNR $\left(\mathrm{Eb} / \mathrm{N}_{0}\right)$. As shown in Fig. 3, we found that when the scan step is no more than 0.01 , the eavesdropper can demodulate the signal correctly. In addition, when $a=1.3$, the demodulated constelation structure was the same as the correct QPSK constelation structure while the information sequence order was entirely incorrect. In this case, there is still a $50 \%$ probability that the entirely wrong decision could be made [28].

In the PM-WFRFT scheme, both branch signals must be demodulated correctly to demodulate the confidential information. So if we chose the scan step $\varepsilon=0.01$, the eavesdropper would need to scan 800 times to search the best parameter with a correct demodulation probability of $25 \%$. In addition, the WFRFT parameter in our scheme is controlled by a pseudo-random sequence and is assumed to be updated every $N$ symbol intervals, which makes it harder to crack the WFRFT parameter. Moreover, only one of nine parameters is utilized to calculate the weight vector while the other parameters are set to zero. If the nine parameters are all used, the system would overwhelm the eavesdropper and the security performance could be even further improved.

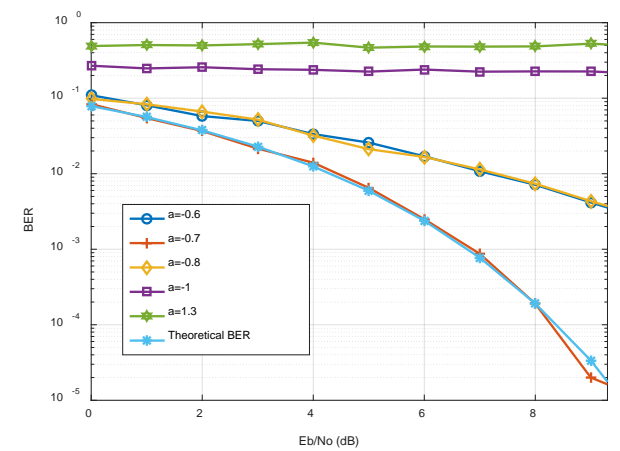

(a)scan step equals 0.1

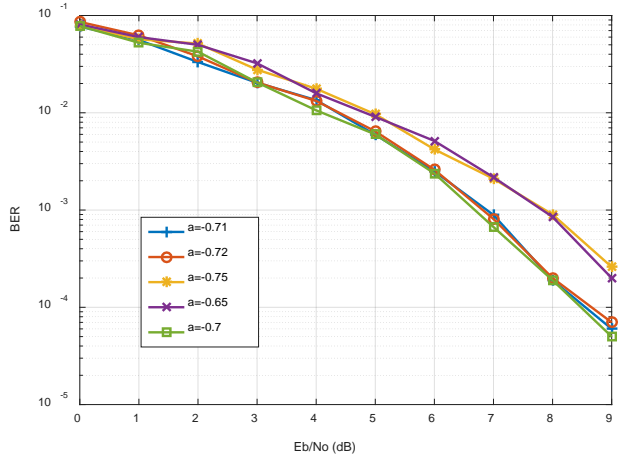

(b) scan step equals 0.01 and 0.05

Fig. 3. BER performance with different scan steps

\section{Depolarization Effect Cancellation and Performance Evaluation}

The PM-based scheme is vulnerable to the depolarization effect, which is mainly presented as PDL and polarization mode dispersion (PMD) [29]. However, PMD only appears in the multi-carrier scenario [26], so we only considered PDL effect in this study. At the receiver side, during the $k$-th symbol interval, the received signal can be denoted as follows:

$$
\mathbf{y}_{m}^{\mathrm{R} k}=\mathbf{H B S}+\mathbf{n}=\left[\begin{array}{ll}
h_{11} & h_{12} \\
h_{21} & h_{22}
\end{array}\right]\left[\begin{array}{l}
b_{m}^{k} \\
1-b_{m}^{k}
\end{array}\right] \mathbf{s}_{m}^{\mathrm{w}}(k)+\left[\begin{array}{l}
n_{1} \\
n_{2}
\end{array}\right], m=1,2,
$$

where $\mathbf{H}$ denotes the satellite channel impulse response matrix, $\mathbf{B}$ denotes the polarization selection vector, $\mathbf{S}$ denotes the transmit WFRFT data and $\mathbf{n}$ is white Gauss noise with spectral density of $N_{0}$.

In satellite scenarios, $\mathbf{H}$ consists of the LOS component $\overline{\mathbf{H}}$ and the non-LOS component $\tilde{\mathbf{H}}$ [30] [31]. In our research, we mainly consider $\overline{\mathbf{H}}$ as the dominating wave propagation mechanism for multipath signals playing a secondary role in satellite communication. And the power of the LOS component is stronger than that of reflections and scattered waves [32], so it can be modeled as follows [33] : 


$$
\overline{\mathbf{H}}=\sqrt{Q}\left[\begin{array}{ll}
\sqrt{1-\beta_{a n t}} & \sqrt{\beta_{a n t}} \\
\sqrt{\beta_{a n t}} & \sqrt{1-\beta_{a n t}}
\end{array}\right]=\sqrt{Q} \mathbf{U} \Sigma \mathbf{V}=\sqrt{Q} \mathbf{U}\left[\begin{array}{cc}
\sqrt{\lambda_{1}} & 0 \\
0 & \sqrt{\lambda_{2}}
\end{array}\right] \mathbf{V},
$$

where $Q$ is the power fading of the non-polarized channel and $\sqrt{\lambda_{m}}, m=1,2$ denotes the eigenvalue. $\mathbf{U}, \mathbf{V}$ are unitary matrixes and $\beta_{\text {ant }}$ is the parameter used to measure the cross polar discrimination (XPD) of the antennas as

$$
X P D=10 \log _{10}\left(\frac{1-\beta_{\text {ant }}}{\beta_{\text {ant }}}\right),
$$

Based on Eq. (21), after inverse WFRFT transformation, Eq. (20) can be rewritten as follows :

$$
\begin{aligned}
& \mathbf{y}_{m}^{\mathrm{R} k}=\left[\begin{array}{ll}
\sqrt{1-\beta_{a n t}} & \sqrt{\beta_{a n t}} \\
\sqrt{\beta_{\text {ant }}} & \sqrt{1-\beta_{a n t}}
\end{array}\right]\left[\begin{array}{l}
b_{m}^{k} \\
1-b_{m}^{k}
\end{array}\right] \hat{s}_{m}^{k}+\left[\begin{array}{l}
\breve{n}_{1} \\
\breve{n}_{2}
\end{array}\right] \\
& =\left[\begin{array}{l}
\sqrt{1-\beta_{a n t}} \hat{s}_{m}^{k}+\breve{n}_{1} \\
\sqrt{\beta_{\text {ant }} \hat{s}_{m}^{k}}+\breve{n}_{2}
\end{array}\right], b_{m}^{k}=0 ;\left[\begin{array}{l}
\sqrt{\beta_{a n t}} \hat{s}_{m}^{k}+\breve{n}_{1} \\
\sqrt{1-\beta_{a n t}} \hat{s}_{m}^{k}+\breve{n}_{2}
\end{array}\right], b_{m}^{k}=1
\end{aligned}
$$

where $\hat{s}_{i}^{k}=\sqrt{Q} s_{i}^{k}, \breve{n}_{1}$ and $\breve{n}_{2}$ are Gauss noise with spectral density of $N_{0}$. According to Eq.(23), at the receiver side, during a symbol period, the signal is received by both two orthogonally polarized antennas and the difference is the signal's power. Therefore, a detection method based on the received signal's power is proposed to distinguish the polarization characteristic of the transmitting antenna.

In order to demodulate the bit $b_{i}^{k}$, at first, during the $k$-th symbol period, the received signal in two symbol time is assumed to be $\mathbf{y}_{1}^{\mathrm{Rk}}$ and $\mathbf{y}_{2}^{\mathrm{Rk}}$, respectively. Then the power ratio is calculated as follows:

$$
P o_{1}^{k}=\frac{\left\|\mathbf{y}_{1}^{\mathrm{Rk}}(1)\right\|^{2}}{\left\|\mathbf{y}_{1}^{\mathrm{Rk}}(2)\right\|^{2}},
$$

The estimator of the bit $b_{1}^{k}$ is:

$$
\Lambda_{1}^{k}=\left[1+\operatorname{sign}\left(\log \left(P o_{1}^{k}\right)\right)\right] / 2,
$$

If the estimator is greater than 1 , it is more likely that $y_{1}^{k}=\mathbf{y}_{1}^{\mathrm{Rk}}(1), b_{1}^{k}=0$; otherwise, $y_{1}^{k}=\mathbf{y}_{1}^{\mathrm{R} k}(2), b_{1}^{k}=1$. The same method can be used to obtain $b_{2}^{k}$. After both components of the polarized signal are obtained, based on Eq. (26), the $k$-th symbol's PS is demodulated. Then according to the Maximum Likelihood (ML) decision rule, the polarization constellation point $\mathbf{P}_{k}$ can be classified from $\mathbf{P}_{k}^{\mathrm{R}}$. The confidential information can be recovered once all constellation points have been classified.

$$
\gamma_{k}^{\mathrm{R}}=\arctan \left(\frac{\operatorname{abs}\left(y_{2}^{k}\right)}{\operatorname{abs}\left(y_{1}^{k}\right)}\right), \eta_{k}^{\mathrm{R}}=\Xi\left(y_{2}^{k}\right)-\Xi\left(y_{1}^{k}\right),
$$

where $\Xi(\bullet)$ is the phase acquisition operation. For the QAM signal demodulation, firstly, $\left\{y_{1}^{k}\right\}_{k=1}^{K}$ and $\left\{y_{2}^{k}\right\}_{k=1}^{K}$ are combined to obtain the diversity gain. Then the combined signals are demodulated according to the QAM demodulation rule to recover $\mathrm{I}_{q}$. Finally, the whole information sequence can be recovered from $\mathrm{I}_{p}, \mathrm{I}_{q}$, and $\mathrm{I}_{b}$. 
Notably, the dual components of the transmit signal are different, so if the eavesdropper does not know the confidential information is carried by the signal's PS, two orthogonal polarizations will be considered as a case of simple spatial modulation like in [20]. From this point of view, the security performance is further enhanced by this process. After the PS of the transmit signal is obtained, the symbol error rate (SER) of the PM branch can be derived as follows:

$$
\begin{aligned}
& P_{S E R}=\frac{1}{M} \sum_{i=1}^{M} S E R_{i}, \\
& S E R_{i}=\sum_{j=1}^{c} 2\left[\int_{\psi_{i j}}^{\pi} \int_{0}^{\alpha\left(\vartheta_{i j}, \psi_{i j}\right)} f\left(t_{i}, \varphi_{i}\right) \mathrm{d} \varphi_{i} \mathrm{~d} t_{i}+\int_{\vartheta_{i j}}^{\psi_{i j} \alpha\left(\vartheta_{j}, t_{i}\right)} \int_{0}^{2} f\left(t_{i}, \varphi_{i}\right) \mathrm{d} \varphi_{i} \mathrm{~d} t_{i}\right],
\end{aligned}
$$

where $\alpha\left(\vartheta_{i j}, t_{i}\right)=\arccos \left(\tan \vartheta_{i j} / t_{i}\right), f\left(t_{i}, \varphi_{i}\right)$ is the joint probability density function of $P_{i}^{\mathrm{R}}$ 's co-latitude $t_{i}$ and azimuth $\varphi_{i}$ with additional AWGN noise, which can be expressed as follows:

$$
f\left(t_{i}, \varphi_{i}\right)=\frac{\sin t_{i}}{4 \pi} e^{-E_{i}^{\mathrm{R}}\left(1-\cos t_{i}\right) /\left(2 N_{0}\right)}\left[1+E_{i}^{\mathrm{R}}\left(1+\cos t_{i}\right) /\left(2 N_{0}\right)\right],
$$

where $\varphi_{i}$ and $t_{i}$ are co-latitude and azimuth of $P_{i}^{\mathrm{R}}$ and the received signal to noise ratio (RSNR) is $\xi=E_{i}^{\mathrm{R}} / N_{0}$, which can be calculated as follows:

$$
E_{i}^{\mathrm{R}}==\left|y_{1}^{i}\right|^{2}+\left|y_{2}^{i}\right|^{2}=\left|\sqrt{1-\beta_{\text {ant }}} \hat{s}_{1}^{i}\right|^{2}+\left|\sqrt{1-\beta_{\text {ant }}} \hat{s}_{2}^{i}\right|^{2} \leq E_{i}^{\mathrm{T}}=\left(s_{2}^{i}\right)^{2}+\left(s_{1}^{i}\right)^{2}
$$

where $E_{i}^{\mathrm{T}}$ is the transmitting power. $\vartheta_{i j}$ denotes half of the adjacent constellation sphere distance and $\psi_{i j}$ denotes the sphere distance between $P_{i}^{\mathrm{R}}$ and the endpoints of its decision region ( $c$ is the number of endpoints):

$$
\begin{aligned}
& \vartheta_{i 1}=\vartheta_{i 2}=\arccos \left[\cos ^{2}\left(2 \gamma_{i}^{\mathrm{R}}\right)+\sin ^{2}\left(2 \gamma_{i}^{\mathrm{R}}\right) \cos O\right], \\
& \psi_{i 1}=2 \gamma_{i}^{\mathrm{R}}, \psi_{i 2}=\pi-2 \gamma_{i}^{\mathrm{R}}, k=2,
\end{aligned}
$$

where $i=1,2, \cdots, M_{P}$ and $O$ is the spheric angle between the adjacent polarization constellation points :

$$
O=\angle P_{i}^{R} P_{\mathrm{H}} P_{j}^{R}=2 \arcsin \left[\sin \left(d_{\mathrm{T}} / 2\right) / \sin \left(2 \gamma_{i}^{R}\right)\right],
$$

where $d_{\mathrm{T}}$ is the original spherical constellation distance and $P_{\mathrm{H}}$ is the horizontal PS.

\section{Experimental Classification Results and Analysis}

In this section, we discuss the security transmission performance and robustness to PDL effect of the PM-WFRFT scheme. We first examined the LPD influence on the TM signal's PSD after performing PM; we also measured the similarity between the distribution of the WFRFT signal and Gaussian distribution.

The average of 50 simulation results is shown here to provide an example of the PSD curve. Two random bit sequences are generated and modulated into 1024 QPSK symbols and 4PM symbols, respectively, where the number before PM represents the modulation order. QPSK signals are used as the TM signals and 4PM signals are used to produce the weight vectors. The WFTFR signals are obtained based on Eq.(4). The normalized PSD of the QPSK signal, $\mathbf{s}_{1}^{\mathrm{w}}$ and $\mathbf{s}_{2}^{\mathrm{w}}$ are shown in Fig. $\mathbf{4}$, it is found that the PSD are nearly the same, this is because 
the PSD did not change after being processed by WFRFT; further, the designed PM constellation does not impact the PSD of the TM signal (as analyzed in Section 2.1). Notice that since the means of the random sequences are not equal to zero, there are irregular waves on the envelope as shown in Fig. 4. Furthermore, we have to point out that, for other TM modulation techniques, whose constellation mean is zero can also be applied here.

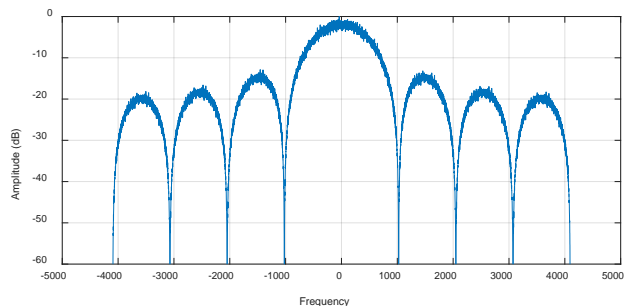

(a) PSD of QPSK signal

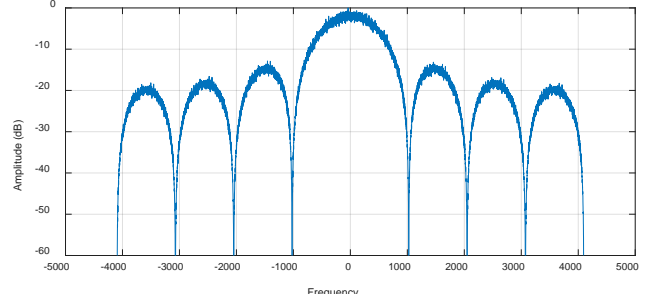

(b) PSD of $\mathbf{s}_{1}^{\mathrm{W}}$ based on eq. (4)

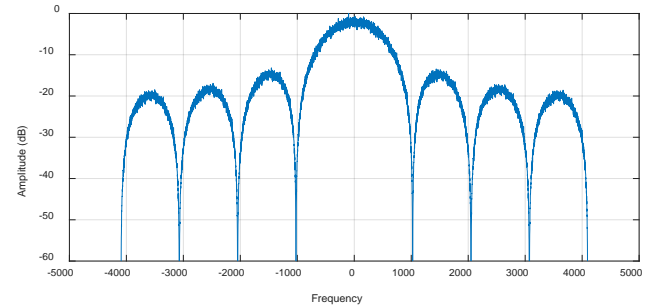

(c) PSD of $\mathbf{s}_{2}^{\mathrm{W}}$ based on eq. (4)

Fig. 4. PSD of QPSK, $s_{1}^{w}$ and $s_{2}^{w}$

Next, to draw a quantificational description of the comparability of the WFRFT signal to Gaussian statistics, we investigated the Kurtosis of the WFRFT signal. Based on the analysis in [17], QPSK and 16QAM were chosen as TM signals in our research and 4PM and 8PM were used to carry confidential information.

The Kurtosis of the WFRFT signal's real and imaginary parts are respectively given in Fig. 5. We find them to be almost identical, because the constellation structure is symmetrical to the origin of the coordinate. Comparing Fig.5(a)-(b) with (c)-(f), it is found that the distribution is more Gaussian when combined with PM. In addition, by comparing Fig. 5(a), 5(c), and 5(e) with 5(b), 5(d), and 5(f), it is found that the curves in the latter are closer to zero because the modulation order of the TM signal is higher. These results conform to the analysis in Section 2.1 that with the same parameter $a$, higher modulation order technique results in closer similarity to Gaussian statistics. Therefore, by appropriately selecting the parameter $a$, the modulated signal can be transformed into the quasi-Gaussian signal which is difficult to detect.

Finally, we investigate the SER performance of the PM-WFRFT scheme versus PDL to determine the robustness of the PM-WFRFT scheme against the PDL effect. We fix the RSNR to $15 \mathrm{~dB}$ and define the PDL as $P D L=10 \log 10\left(\lambda_{1} / \lambda_{2}\right)$. Fig. 6 shows the SER curves of the PM-WFRFT scheme and the PM scheme provided by authors in [19], where the number before the abbreviation represents the modulation order. We find that the SER performance of PM deteriorates more severely than that of PM-WFRFT as PDL increases, because the PM constellation structure is distorted and the RSNR attenuates quickly. Conversely, since the two components of the polarized signal are transmitted separately in two symbol periods in the PM-WFRFT scheme, there is no mutual interference between the orthogonally polarized components. However, as shown in Eq. (29), the RSNR decreases as PDL increases, which 
also causes SER performance degradation. Regardless, the proposed scheme still well outperforms the PM scheme in regards to robustness to PDL effect.

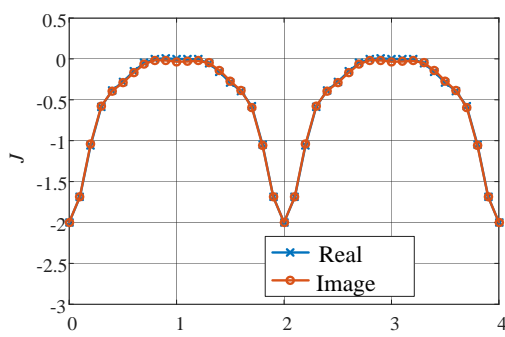

(a) QPSK

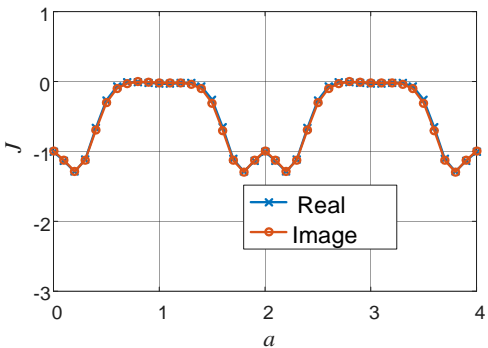

(c) QPSK with 4PM

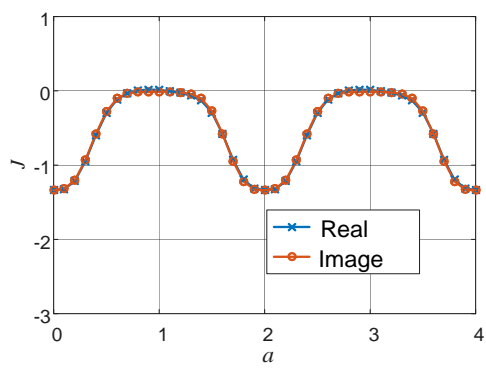

(e) QPSK with 8PM

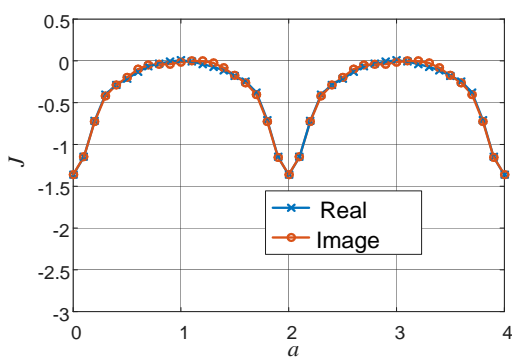

(b) 16QAM

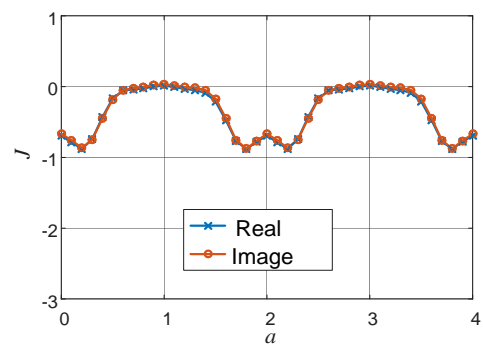

(d) 16QAM with 4PM

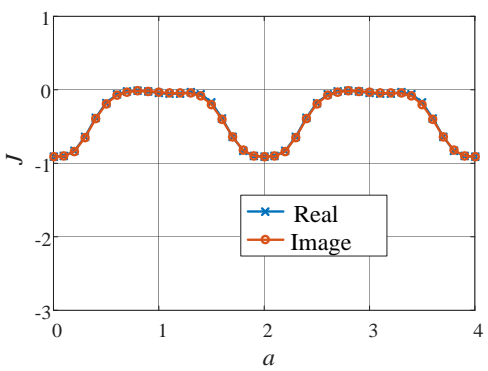

(f) 16QAM with 8PM

Fig. 5. Kurtosis of WFRFT signals versus $a$

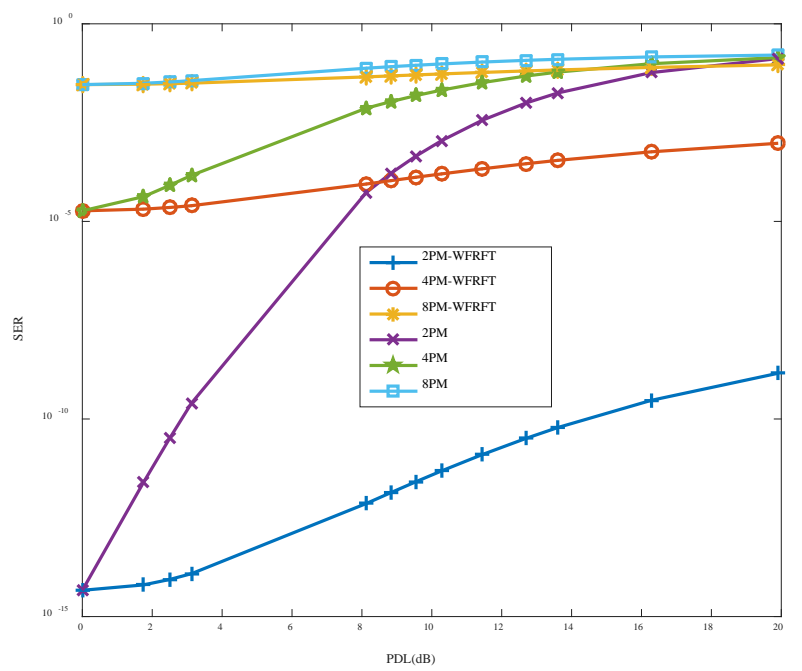

Fig. 6. SER performance versus PDL 


\section{Conclusion}

In this paper, we propose a physical layer security scheme based on polarization modulation and weighted fractional Fourier transform (PM-WFRFT) techniques. With the proposed scheme, the distribution of the transmit signal is similar to the Gauss distribution, which makes it hard to be detected. In condition, the WFRFT parameter is controlled by a pseudo-random sequence and is updated in real time, which make the WFRFT parameter difficult to crack, thus the confidential information is hard to be recovered. On the other hand, the two components of the polarized signal are transmitted in turn to avoid the PDL effect and an effective signal detection method based on the signal's power is proposed.

\section{References}

[1] Z. Gan, P. Arapoglou, and B. Ottersten, "Physical Layer Security in Multibeam Satellite Systems," IEEE Transactions on Wireless Communications, vol. 11, no.2, pp. 852-863, Feburary, 2012. Article (CrossRef Link)

[2] A. Mukherjee, S. A. A. Fakoorian, J. Huang, and A. L. Swindlehurst, "Principles of Physical Layer Security in Multiuser Wireless Networks: A Survey," IEEE Communications Surveys \& Tutorials, vol. 16, no.3, pp. 1550-1573, Feburary, 2014. Article (CrossRef Link)

[3] Q. Z. Ahmed, K. H. Park, and M. S. Alouini, "Ultrawide Bandwidth Receiver Based on a Multivariate Generalized Gaussian Distribution," IEEE Transactions on Wireless Communications, vol. 14, no.4, pp. 1800-1810, November, 2015. Article (CrossRef Link)

[4] M. K. Tsay, C. H. Liao, C. S. Shyn, and T. Y. Yang, "Simultaneous AJ and LPD Evaluations for Secure Communication," in Proc. of MILCOM 2007 - IEEE Military Communications Conference of Conference, pp. 1-6, October 29-31, 2007. Article (CrossRef Link)

[5] L. Zhao, L. Wang, G. Bi, L. Zhang, and H. Zhang, "Robust Frequency-Hopping Spectrum Estimation Based on Sparse Bayesian Method," IEEE Transactions on Wireless Communications, vol. 14, no.2, pp. 781-793, Feburary, 2015. Article (CrossRef Link)

[6] A. D. Wyner, "The wire-tap channel," Bell System Technical Journal, vol. 54, no.8, pp. 1355-1387, October, 1975. Article (CrossRef Link)

[7] J. Lei, H. Zhu, M. A. Vaazquez-Castro, and A. Hjorungnes, "Multibeam SATCOM systems design with physical layer security," in Proc. of IEEE International Conference on Ultra-Wideband (ICUWB) of Conference, pp. 555-559, September 14-16, 2011. Article (CrossRef Link)

[8] N. Zhao, F. R. Yu, M. Li, Q. Yan, and V. C. Leung, "Physical layer security issues in interference-alignment-based wireless networks," IEEE Communications Magazine, vol. 54, no.8, pp. 162-168, August, 2016. Article (CrossRef Link)

[9] N. Zhao, F. R. Yu, M. Li, and V. C. Leung, "Anti-eavesdropping schemes for interference alignment (IA)-based wireless networks," IEEE Transactions on Wireless Communications, vol. 15, no.8, pp. 5719-5732, May, 2016. Article (CrossRef Link)

[10] Z. Luo, H. Wang, and W. Lv, "Directional polarization modulation for secure transmission in dual-polarized satellite MIMO systems," in Proc. of 2016 8th International Conference on Wireless Communications \& Signal Processing (WCSP) of Conference, pp. 1-5, October 13-15, 2016. Article (CrossRef Link)

[11] T. Hong, M. Z. Song, and Y. Liu, "Dual-Beam Directional Modulation Technique for Physical-Layer Secure Communication," IEEE Antennas and Wireless Propagation Letters, vol. 10, no.1, pp. 1417-1420, December, 2011. Article (CrossRef Link)

[12] R. Ma, L. Dai, Z. Wang, and J. Wang, "Secure communication in TDS-OFDM system using constellation rotation and noise insertion," IEEE Transactions on Consumer Electronics, vol. 56, no.3, pp. 1328-1332, October, 2010. Article (CrossRef Link)

[13] W. Trappe, "The challenges facing physical layer security," IEEE Communications Magazine, vol. 53, no.6, pp. 16-20, June, 2015. Article (CrossRef Link) 
[14] W. Dong, L. Lili, Z. Meng, Q. Rong, and H. Weiqing, "A Polarization state Modulation based Physical Layer Security scheme for Wireless Communications," in Proc. of MILCOM 2016 - 2016 IEEE Military Communications Conference of Conference, pp. 1195-1201, November 1-3, 2016. Article (CrossRef Link)

[15] L. Arend, R. Sperber, M. Marso, and J. Krause, "Polarization shift keying over satellite Implementation and demonstration in Ku-band," in Proc. of Advanced Satellite Multimedia Systems Conference and the Signal Processing for Space Communications Workshop of Conference, pp. 165-169, September 8-10, 2014. Article (CrossRef Link)

[16] T. Qi and Y. Wang, "Capacity Analysis of a Land Mobile Satellite System Using Dual-Polarized Antennas for Diversity," in Proc. of 2015 IEEE 82nd Vehicular Technology Conference (VTC2015-Fall) of Conference, pp. 1-5, September 6-9, 2015. Article (CrossRef Link)

[17]L. Mei, X. Sha, Q. Ran, and N. Zhang, "Research on the application of 4-weighted fractional Fourier transform in communication system," Science China Information Sciences, vol. 53, no.6, pp. 1251-1260, June, 2010. Article (CrossRef Link)

[18] S. Benedetto and P. T. Poggiolini, "Multilevel polarization shift keying: optimum receiver structure and performance evaluation," IEEE Transactions on Communications vol. 42, no.234, pp. 1174-1186, Mar, 1994. Article (CrossRef Link)

[19] W. Dong, Z. Meng, F. Wei, and H. Weiqing, "A spectrum efficient polarized PSK/QAM scheme in the wireless channel with polarization dependent loss effect," in Proc. of 2015 22nd International Conference on Telecommunications (ICT), Sydney, NSW, Australia of Conference, pp. 249-255, April 27-29, 2015. Article (CrossRef Link)

[20] P. Henarejos and A. Perez-Neira, "Dual Polarized Modulation and Reception for Next Generation Mobile Satellite Communications," IEEE Transactions on Communications, vol. 63, no.10, pp. 3803-3812, July, 2015. Article (CrossRef Link)

[21] C. Li, H. J. Yang, F. Sun, J. M. Cioffi, and L. Yang, "Multiuser Overhearing for Cooperative Two-Way Multiantenna Relays," IEEE Transactions on Vehicular Technology, vol. 65, no.5, pp. 3796-3802, June, 2016. Article (CrossRef Link)

[22] C. Li, S. Zhang, P. Liu, F. Sun, J. M. Cioffi, and L. Yang, "Overhearing Protocol Design Exploiting Intercell Interference in Cooperative Green Networks," IEEE Transactions on Vehicular Technology, vol. 65, no.1, pp. 441-446, January, 2016. Article (CrossRef Link)

[23] C. Li, F. Sun, J. M. Cioffi, and L. Yang, "Energy efficient MIMO relay transmissions via joint power allocations," IEEE Transactions on Circuits and Systems II: Express Briefs, vol. 61, no.7, pp. 531-535, May, 2014. Article (CrossRef Link)

[24] C. Li, H. J. Yang, F. Sun, J. M. Cioffi, and L. Yang, "Approximate closed-form energy efficient PA for MIMO relaying systems in the high SNR regime," IEEE Communications Letters, vol. 18, no.8, pp. 1367-1370, June, 2014. Article (CrossRef Link)

[25] L. Mei, Q. Zhang, X. Sha, and N. Zhang, "WFRFT Precoding for Narrowband Interference Suppression in DFT-Based Block Transmission Systems," IEEE Communications Letters, vol. 17, no.10, pp. 1916-1919, September, 2013. Article (CrossRef Link)

[26] J. G. Proakis, Digital communications: McGraw-Hill, 2001. Article (CrossRef Link)

[27] R. Li, F. Liu, Z. Zeng, and C. Feng, "A M-Continuous-Polarization Modulation scheme for improving the bandwidth efficiency," in Proc. of 2014 International Symposium on Wireless Personal Multimedia Communications (WPMC) of Conference, pp. 253-258, September 7-10, 2014. Article (CrossRef Link)

[28] F. Xiaojie, S. Xuejun, and L. Yue, "MP-WFRFT and constellation scrambling based physical layer security system," China Communications, vol. 13, no.2, pp. 138-145, Feburary, 2016. Article (CrossRef Link)

[29] W. Dong, F. Chunyan, and G. Caili, "An optimal pre-compensation based joint polarization-amplitude-phase modulation scheme for the power amplifier energy efficiency improvement," in Proc. of IEEE International Conference on Communications (ICC) of Conference, pp. 4137-4142, June 9-13, 2013. Article (CrossRef Link)

[30] K. P. Liolis, Go, x, V. mez, x, J., et al., "Statistical Modeling of Dual-Polarized MIMO Land Mobile Satellite Channels," IEEE Transactions on Communications, vol. 58, no.11, pp. 3077-3083, 
September, 2010. Article (CrossRef Link)

[31] M. Yofune, J. Webber, K. Yano, H. Ban, and K. Kobayashi, "Optimization of Signal Design for Poly-Polarization Multiplexing in Satellite Communications," IEEE Communications Letters, vol. 17, no.11, pp. 2017-2020, October, 2013. Article (CrossRef Link)

[32] A. Knopp, R. T. Schwarz, D. Ogermann, C. A. Hofmann, and B. Lankl, "Satellite System Design Examples for Maximum MIMO Spectral Efficiency in LOS Channels," in Proc. of 2008 IEEE Global Telecommunications Conference of Conference, pp. 1-6, Nov. 30-Dec. 4, 2008.

Article (CrossRef Link)

[33] Y. Yang, X. Xin, J. Bin, and G. Xiqi, "An adaptive coding method for dual-polarized mobile satellite communications," in Proc. of 2014 Sixth International Conference on Wireless Communications and Signal Processing (WCSP), Hefei, China of Conference, pp. 1-5, October 23-25, 2014. Article (CrossRef Link)
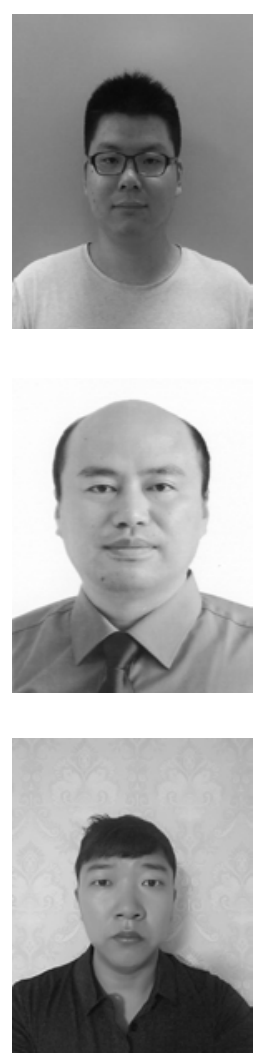

Zhangkai Luo, received the B.Eng. degree in communication engineering from Hebei University of Technology, Tianjin, China, in 2011. Currently he is a research student working towards the Ph.D. degree in PLA University of Science and Technology, China. His research interests include Information processing and confrontation.

Huali Wang, received the Ph.D. degree in electronic engineering in 1997 from Nanjing University of Science and Technology, China. He is currently a professor at Nanjing Institute of Communication Engineering, PLA University of Science and Technology, China. His research interests include digital signal processing, array Signal Processing, sparse signal sampling

Kaijie Zhou, received the B.Eng. degree in electrical engineering and automation from Hefei University of Technology, Hefei, China, in 2007. Currently he is a research student working towards the Ph.D. degree in PLA University of Science and Technology, China. His research interests include Information processing and confrontation. 